\title{
DIVERTÍCULOS VESICALES CONGÉNITOS EN LA INFANCIA Y REFLUJO VÉSICO- URETERAL
}

\author{
José María Garat, Oriol Angerri, Jorge Caffaratti y Pietro Moscatiello.
}

Unidad de Urología Pediátrica. Servicio de Urología. Fundació Puigvert. Barcelona. España.

\begin{abstract}
Resumen.- OBJETIVO: Analizar nuestra serie de divertículos congénitos primarios (DVCP) y su relación con el reflujo vésicoureteral.
\end{abstract}

MÉTODOS: En 10 años hemos asistido a 23 niños con DVCP. Once eran divertículos grandes (más de $2 \mathrm{~cm}$ de diámetro) y doce eran pequeños. En el primer grupo, 4 niños tenían reflujo vésico-ureteral y en el segundo cinco. En el grupo $A$ se reimplantaron los uréteres refluyentes al extirpar el divertículo. En el grupo B no se operó en ningún caso el divertículo o el reflujo.

RESULTADOS: Analizaremos por separado los resultados de ambos grupos. Grupo A: fueron operados, extirpándose el/ los divertículo/s. Sólo recidivaron los divertículos en el caso de síndrome de Ehlers-Danlos. En todos los casos reimplantados no hubo reflujo postoperatorio. Sin embargo, apareció un reflujo post-cirugía en un caso de vejiga polidiverticular, a la que se le extirparon varios divertículos sin reimplante. Grupo B: los divertículos pequeños no fueron operados. La evolución espontánea del reflujo fue similar a la de la población general sin divertículos.

CONCLUSIONES: Los divertículos vesicales se asocian con frecuencia a reflujo vésico-ureteral. La presencia del reflujo no constituye, en el momento actual, un condicionante absoluto del tratamiento quirúrgico o endoscópico. Cuando los divertículos son grandes (Grupo A), la indicación quirúrgica está dada por las infecciones recidivantes, los trastornos miccionales y no por la presencia de reflujo. Si se operan, se reimplanta el uréter porque tiene reflujo o porque hay razones técnicas de debilidad parietal. Cuando los divertículos son pequeños (Grupo B), la presencia de reflujo no condiciona el tratamiento, siendo la tasa de resolución espontánea similar a la de la población general.

Palabras clave: Divertículos vesicales. Reflujo vésico-ureteral. Infecciones urinarias. Trastornos miccionales.

Summary.- OBJECTIVES: To analyze our series of primary congenital diverticula (PCD) and their association with vesicoureteral reflux.

METHODS: We have taken care of 23 children with PCD. Eleven of them had big diverticula (> $2 \mathrm{~cm}$ ) and twelve small. In the first group, 4 children had vesicoureteral reflux and 5 in the second group. In group A, ureteral reimplantation was performed at the time of diverticulum excision. Nor diverticula neither refluxes were operated in group $B$.

RESULTS: We analyze separately results in both groups. Group A: Patients were operated including diverticulum excision. There were not recurrences except in one case with Ehler-Danlos 
Syndrome. No reimplanted ureter showed postoperative reflux. Nevertheless, one case with multiple bladder diverticula without reflux presented reflux after the excision of several diverticula without reimplantation.

Group B: Small diverticula did not undergo surgery. Spontaneous outcome of reflux was similar to that of the general population without diverticula.

CONCLUSIONS: Bladder diverticula are frequently associated with vesicoureteral reflux. The presence of reflux is not an absolute condition for surgical or endoscopic treatment. When diverticula are big in size (Group A) the indication for surgery comes from recurrent infection or voiding disorders, not from reflux. If they undergo surgery, ureteral reimplantation is performed in the case they had reflux or for technical reasons like bladder wall weakness. When diverticula are small /Group $B)$ the presence of reflux does not condition treatment, being the rate of spontaneous resolution similar to the general population.

Keywords: Bladder diverticula. Vesicoureteral reflux. Urinary tract infection. Voiding disorders.

\section{INTRODUCCIÓN}

Mucho se ha escrito sobre divertículos vesicales y reflujo vésico-ureteral. La mayoría de trabajos se refieren a los divertículos en general (primarios y secundarios) y eso no contribuye a aclarar el tema. Debemos establecer la notoria diferencia entre divertículos vesicales congénitos primarios (DVCP) y los secundarios a patologías generalmente obstructivas cérvico-uretrales o vejiga neuropática. La misma causa obstructiva que genera el divertículo, puede crear las condiciones favorables para que se desarrolle y/o mantenga el reflujo. En cambio, en los DVCP la presencia de un reflujo puede deberse fundamentalmente a tres situaciones: modificación anatómica creada por el mismo divertículo, trastorno funcional secundario a la presencia del divertículo o simple coincidencia.

Los DVCP son poco frecuentes y en su mayoría únicos. Analizaremos nuestra serie de los últimos diez años, algunos de ellos con reflujo vésico-ureteral y otros sin reflujo. Trataremos de observar el comportamiento de ese reflujo y su relación con los divertículos.

\section{MATERIAL Y MÉTODO}

Desde septiembre de 1995 a septiembre de 2005, hemos asistido a 23 niños con DVCP. Sus edades en el momento del diagnóstico oscilaban entre dos meses y diez años (media 4.2), 18 eran varones y 5 eran niñas.

Los hemos dividido en dos grupos en función del tamaño del o de los divertículos, hecho que para nosotros constituyó un elemento importante para la indicación quirúrgica.
El primer grupo (Grupo A) son 11 casos de divertículos de más de $2 \mathrm{~cm}$ de diámetro. Todos fueron operados por vía abierta menos uno.

En cambio en el grupo de divertículos pequeños (Grupo B) ninguno fue intervenido quirúrgicamente.

\section{GRUPO A}

En el grupo A, diez eran varones y una niña.

El motivo de consulta más frecuente fue infección urinaria febril (5 casos); 3 niños consultaron por infecciones urinarias de repetición, uno por síndrome enurético, uno por hematuria macroscópica y uno por dolor en fosa ilíaca derecha. Uno tenía un síndrome de EHLERS-DANLOS.

El diagnóstico se efectuó mediante cistografía retrógrada y miccional en todos los casos, aunque fue sospechado previamente por ecografía en cuatro. Todos los divertículos medían más de $2 \mathrm{~cm}$ de diámetro, no estando reseñado en la mayoría el tamaño vesical, para poder establecer la relación de tamaño diverticular/tamaño vesical.

El divertículo era único en 7 pacientes, doble en dos casos y en dos se trataba de una vejiga multidiverticular, sin ningún elemento obstructivo.

Se acompañaban de reflujo vésico-ureteral unilateral en 3 casos (GI y GII) y de reflujo bilateral $G$ III en un caso.

En cuanto a la topografía, eran en su gran mayoría posterolaterales (7); uno era paraureteral bilateral y dos vejigas eran multidiverticulares.

Se realizó estudio urodinámico en cuatro casos, para descartar la hiperactividad del detrusor u obstrucción por disinergia: dos estudios fueron normales y en el caso de la vejiga multidiverticular se descartó obstrucción infravesical, pero se informó de detrusor hipocontráctil. Un paciente tenía flujo miccional "aplanado".

En nueve pacientes se realizó uretrocistoscopia preoperatoria que, en todos los casos, nos permitió observar la o las bocas diverticulares, a la vez que pudimos certificar la normalidad del canal uretral y del cuello vesical. La clasificación topográfica se completó observando el orificio diverticular.

Diez niños fueron operados; uno antes de consultar en este Centro (era el paciente con síndrome de EHLERSDANLOS). Se decidió observación clínica en un lactante de 9 meses.

En la cirugía la vía empleada fue la intra y extravesical, resecándose el o los divertículos. En el paciente con vejiga multidiverticular no se extirparon la totalidad de los divertículos, sino sólo los cinco más grandes de un total de nueve. El paciente con síndrome de EHLERS-DANLOS fue operado fuera de nuestra Institución. 
En 5 casos se realizó reimplante urétero-vesical, tipo Cohen. En 4 casos (5 uréteres) existía reflujo y en el restante la disección del uréter muy adosado a la pared diverticular, dejó su inserción en vejiga en unas condiciones que posiblemente favorecerían el reflujo post-operatorio.

En la vejiga multidiverticular no se realizó reimplante ureteral. Tampoco lo hicieron en el síndrome de EHLERS-DANLOS.

\section{GRUPO B}

En el grupo B, de divertículos pequeños, menores de $2 \mathrm{~cm}$ de diámetro, hay 12 casos. El predominio masculino no era tan evidente: 8 eran varones y 4 niñas.

La mayoría consultó por ITU febril (6 casos). Cuatro casos fueron diagnosticados cistográficamente en un estudio por diagnóstico prenatal de ectasia renal. Dos niñas fueron estudiadas urodinámicamente por síndrome enurético.

El diagnóstico se efectuó mediante cistografía retrógrada y miccional en todos los casos.

Había sospecha de anomalía de aparato urinario superior por ecografía prenatal en cuatro casos y ecografía postnatal en dos, con ectasia unilateral. En ningún caso se sospechó divertículo.

Se acompañaban de reflujo vésico-ureteral unilateral en cinco casos (4 grado II y 1 grado III).

La topografía no pudo ser precisada como en el grupo anterior, ya que en ningún caso se realizó cistoscopia.

En tres casos se realizó estudio urodinámico, que demostró hiperactividad del detrusor. Las flujometrías fueron normales.

Ningún niño de este grupo fue intervenido quirúrgicamente, ya sea por vía abierta o endoscópicamente por reflujo.

\section{RESULTADOS}

\section{GRUPO A}

Los 10 casos operados tuvieron una evolución favorable (seguimiento de 2 a 20 años, media 9.3 años) El niño de 9 meses sigue en observación por 5 años. No tuvimos ninguna recidiva del o los divertículos $y$, en todos los reimplantados, no hubo reflujo vésico-ureteral postoperatorio (Figura 1).

En el niño con síndrome de EHLERS-DANLOS (operado en otro hospital) se extirparon algunos divertículos a pesar de lo cual, la cistografía actual es muy similar a la pre-operatoria (¿́recidiva?)

El caso de la vejiga multidiverticular es especial: el niño, con severos trastornos miccionales preoperatorios, normalizó sus micciones y la urodinámica mostró una "recuperación" de la capacidad contráctil del detrusor. Sin embargo, apareció reflujo Gll bilateral. Inicialmente estuvo en quimioprofilaxis. Los divertículos que no fueron extirpados no crecieron. Ahora lleva nueve años de seguimiento, asintomático.

Otro niño que preoperatoriamente tenía flujo "aplanado" y refería chorro miccional sin fuerza, normalizó postoperatoriamente su flujometría y sus micciones.

En 7 casos se realizó anatomía patológica del divertículo extirpado, encontrándose en todos fibras musculares, aunque en la mayoría eran muy tenues, sobre todo en la cúpula del divertículo.

\section{GRUPO B}

Ningún caso fue operado. Fueron puestos en quimioprofilaxis nueve niños (los cinco con reflujo más otros cuatro que habían tenido infecciones urinarias repetidas) Tres niños se trataron con anticolinérgicos (tolterodina 4 $\mathrm{mg} /$ día) entre cinco y ocho meses.

De los cinco niños que tenían reflujo vésico-ureteral, éste desaparció en cuatro casos, con un tiempo de evolución entre seis y veinticinco meses. Ninguno presentó nuevos episodios de infección urinaria febril. Un caso persiste en quimioprofilaxis, sin infección. Las dos niñas con síndrome enurético se curaron clínicamente entre uno y tres años.

\section{DISCUSIÓN}

Se trata de dos Grupos claramente diferentes en su presentación, en su tratamiento y en su evolución, motivo por el cual, los analizaremos por separado.

\section{GRUPO A}

Los pacientes del Grupo A tienen en común el haber sido tratados quirúrgicamente (menos uno). Gracias a

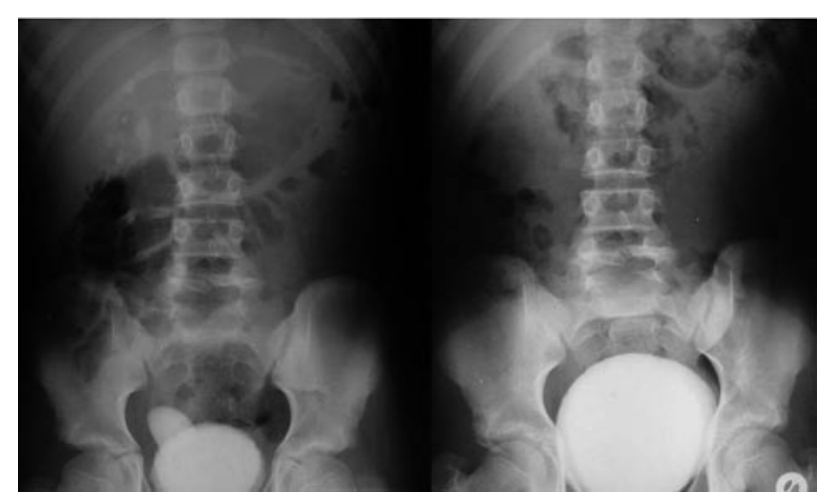

FIGURA 1. Divertículo póstero-lateral más reflujo vésico-ureteral. Cistografía pre y postoperatoria. 
la extirpación de los divertículos, hemos observado que en su pared había en los siete casos en los que se realizó anatomía patológica: fibras musculares distendidas, constituyendo una fina capa. Esto contradice algo la definición que clásicamente se hace de divertículo: hernia de la mucosa a través de la pared muscular (1). La existencia de esta tenue capa de todos modos no tiene una capacidad contráctil capaz de vaciar hacia la vejiga adecuadamente el divertículo y, sobre todo, cuando son grandes, queda siempre un residuo intradiverticular (1).

Forsythe y Smythe (2) en 1959 y sobre todo Johnston (3) en 1960, fueron los primeros en describir los divertículos primarios en niños sin obstrucción. Desde entonces hay que diferenciar claramente los divertículos primarios de los secundarios a válvulas de uretra posterior, vejiga neurógena, etc, cuyo tratamiento debe preceder al de los divertículos.

Zia-ul-miraj (4) enfatizó que los divertículos secundarios son auténticas hernias de la mucosa vesical. Tanto los divertículos secundarios como los primarios se han observado en fetos y en recién nacidos (1) por lo que ambos tipos podrían recibir el apelativo de congénitos.

En los DVCP hay un gran predominio masculino como se refleja también en nuestros casos (10 varones-1 niña). La edad más frecuente del diagnóstico es entre los 3 a 7 años $(1,5)$ y generalmente, son un hallazgo ecográfico o radiológico en el estudio de una infección urinaria (6).

Dos teorías han intentado explicar su producción. Unos se producirían por pulsión y hernia $(1,5)$. Esto explicaría su gran predominio masculino pues los varones orinan con más altas presiones, aún intra-útero. Stephens (5) y Williams (3) piensan que el fallo muscular es primitivo. Linke y cols. (7) sostienen que los DVCP se desarrollan en puntos de fusión muscular incompleta como la unión entre el trígono y la cúpula vesical, entidades de origen embriológico diferente. $\operatorname{DVCP}(1,5)$ :

Podemos clasificar por su topografía, tres tipos de

\section{A - Posterolaterales}

De presentación aproximadamente en un $10 \%$.

No están en relación con los orificios ureterales y no suelen asociarse a reflujo vésico-ureteral. Sin embargo pueden crecer mucho y llegar a comprimir la uretra.

\section{B - Paraureterales}

Serían la mayoría $(90 \%)$ Están en relación con el hiato ureteral que constituye el cuello del divertículo. Dentro de éstos hay dos subgrupos según la topografía del meato ureteral: uréter en vejiga (se asocia a reflujo), uréter dentro del divertículo (se asocia a reflujo de alto grado y a displasia renal) (8)

\section{C - Vejigas multidiverticulares (sin obstrucción)}

- Aislados (más frecuentes en niñas)

- Asociadas a síndromes: "Prune belly", Ehlers-Danlos, Williams-Beuren, Menkes, etc.
La clínica de los DVCP puede ser muy variada. Los hay completamente asintomáticos. Frecuentemente se asocian a infecciones urinarias recurrentes. Pueden causar obstrucción a nivel del cuello vesical en el varón (6-9-1011). Esta situación (12-13) se presenta en el $80 \%$ de los casos en el primer año de vida $150 \%$ en los primeros tres meses).

En cuanto a su asociación con el reflujo vésicoureteral, varía mucho de una serie a otra. En nuestros 11 casos del Grupo A, cuatro se asociaban a RVU. Generalmente, son los divertículos paraureterales y esta situación condiciona a veces el tratamiento. Pueden desarrollar una litiasis en su interior (6).

En otras ocasiones, como en dos de nuestros casos, presentan trastornos miccionales con flujo bajo ("aplanado"). Esto puede ser debido a disinergia vésicoesfinteriana no neurógena (en este caso serían divertículos secundarios) (14) y en otras se trataría de un fenómeno observado por nosotros muy claramente en dos de nuestros casos: vejiga polidiverticular y un caso de divertículo de $5 \mathrm{~cm}$ de diámetro. Los varones se presentaron con disuria y chorro miccional muy débil. Flujometría "aplanada" no obstrucción urinaria (urodinamia, uretroscopia) La explicación es la siguiente: el detrusor se contrae pero la orina entra con más facilidad en el o los divertículos que en el cuello vesical, simulando una hipocontractibilidad del detrusor. Al corregirse los divertículos, el paciente, sin más, retoma un ritmo miccional normal, flujo normal y ausencia de residuo postmiccional. Se trata de una fuga de presiones que hemos denominado "reflujo vésico-diverticular" (Figura 2).

Otras manifestaciones clínicas pueden ser: hematuria, como en uno de nuestros casos o dolor en fosa ilíaca (más raro).

Una entidad diferente son las vejigas multidiverticulares en los síndromes de Ehlers-Danlos, Menkes, Williams-Beuren, etc. (1-14-17). La gran mayoría han sido descritos en varones, como nuestro caso. Jorion y cols. (18) describen un caso en una niña con síndrome de EhlersDanlos.

El mecanismo de su producción sería por una debilidad congénita de las fibras musculares del detrusor, vinculada a la enfermedad congénita del colágeno. En estos casos, se preconiza la abstención quirúrgica, salvo excepciones, ya que la recidiva de los divertículos es casi la regla.

El diagnóstico de DVCP se puede sospechar con ecografía (19) (Figura 3).

La cistografía de relleno y miccional es el método más utilizado (1-6-8-20-21). Es importante realizar proyecciones oblicuas para topografiar mejor el divertículo. En ocasiones, sólo se observa en placas miccionales o postmiccionales (11-21).

Se considera que un divertículo vesical es grande si representa más de un tercio del diámetro vesical (9-22). 
Todos nuestros divertículos grandes los hemos agrupado en el Grupo A (más de $2 \mathrm{~cm}$ de diámetro).

El estudio urodinámico sería importante realizarlo (cistometría y flujometría) pre y postoperatoriamente. En la mayoría de nuestros casos lo realizamos y nos fue de utilidad, sobre todo para descartar patología obstructiva o funcional asociada.

El tratamiento de los DVCP grandes es quirúrgico para prevenir o tratar complicaciones y para mejorar en algunos casos la micción del niño. La extirpación solucionará la obstrucción infravesical compresiva cuando existe, evitará el residuo postmiccional y así prevendrá la recidiva de infecciones urinarias. La supresión del divertículo evita la "fuga de presiones" y mejora el vaciado vesical.

\section{GRUPO B}

Se trata de un grupo de niños en los que el o los divertículos, son un hallazgo y no constituyen un elemento patológico que modifique nuestra conducta terapéutica. Son todos pequeños (menos de $2 \mathrm{~cm}$ de diámetro). No fue posible precisar la topografía (no cistoscopia) pero por la radiología parecen ser paraureterales.

En lo que respecta al reflujo, nuestra actuación fue similar a la que realizamos en ausencia del divertículo, es decir, quimioprofilaxis y aguardar la evolución. En la mayoría de los casos (4/5), el reflujo desapareció espontáneamente.

Lo mismo sucedió con la hiperactividad del detrusor con el tratamiento anticolinérgico.

Aunando ambos grupos, podemos pensar que, salvo en casos de desembocadura ureteral claramente intradiverticular y reflujo de alto grado, el reflujo vésico-ure-

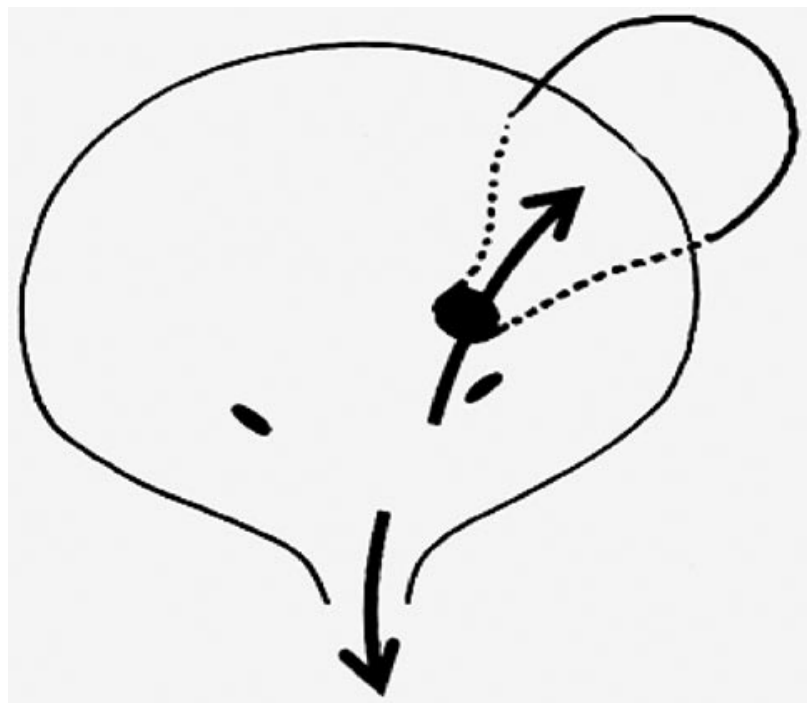

FIGURA 2. Reflujo vesico-diverticular. teral no constituye una clara indicación para el tratamiento quirúrgico o endoscópico.

Afshar y cols. (22) analizaron la evolución de los RVU asociados a DVCP (84 pacientes) comparándolos con una serie similar sin divertículos (95 pacientes). Concluyeron que no había diferencias evolutivas entre ambos grupos y la tasa de resolución espontánea era similar. Se referían a divertículos que por su tamaño no requerían intervención quirúrgica. Piro y cols. (21) en su larga serie de 47 DVCP, indicaban el tratamiento por su tamaño y por la presencia de reflujo (45\%) pero en el momento actual, el tratamiento del RVU es más conservador y muchos de esos casos no tendrían indicación quirúrgica en el momento actual.

También hemos de considerar, como sucedió en uno de nuestros casos (vejiga polidiverticular), la posibilidad de aparición de un reflujo secundaria a la diverticulectomía (en un caso sin reimplante). La buena evolución posterior de este caso, nos reafirma en nuestra postura conservadora.

Queda además la instancia del tratamiento endoscópico que puede ser realizado, si está indicado, en la mayoría de casos con reflujo, a pesar de la presencia del divertículo. Sólo no sería posible en caso de desembocadura intradiverticular del uréter.

La extirpación del o los divertículos puede hacerse por vía abierta o laparoscópica (7). Kok y cols. (23) citan la primera diverticulectomía laparoscópica en un niño de 6 años.

Yu (24) describió una técnica abierta extravesical para tratar simultáneamente la extirpación del divertículo y el reimplante ureteral. Según Sariham y Abes (25) no han de ser operados los divertículos pequeños y asintomáticos y los asociados a síndrome de Ehlers-Danlos.

Extirpado el o los divertículos, las recidivas son excepcionales (26).

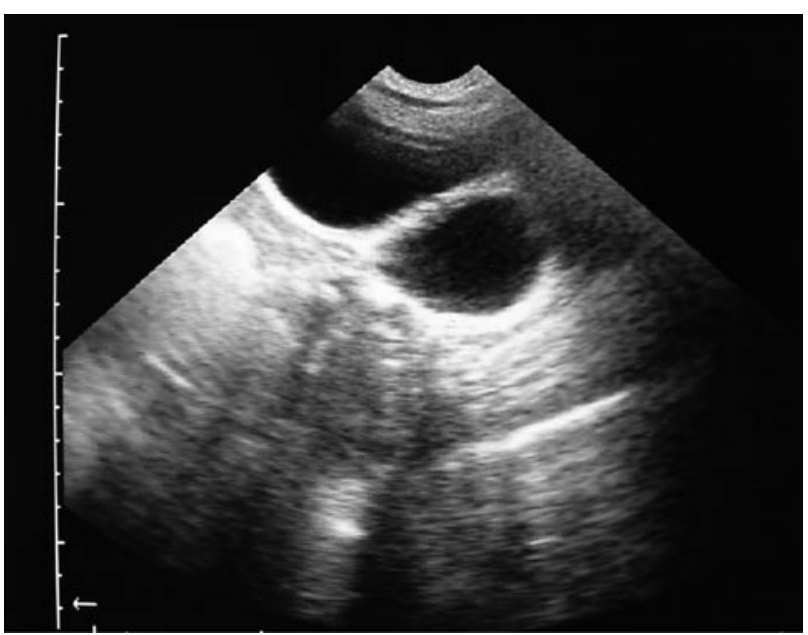

FIGURA 3. Diagnóstico ecográfico. 


\section{BIBLIOGRAFÍA Y LECTURAS RECOMENDADAS (*lectura de interés $y^{* *}$ lectura fundamental)}

1. GARAT, J.M.: “Malformaciones vesicales". Urología Pediátrica (J.M. Garat, R. Gosálbez). pp. 287-313 Salvat, Barcelona, 1987.

2. FORSYTHE, I.; SMYTE, B.: "Diverticulum of the bladder in children. A study of 13 cases". Pediatrics, 24: 322, 1959.

*3. JOHNSTON, J.: "Vesical diverticula without urinary obstruction in childhood". J. Urol., 84: 535, 1960.

4. ZIA-UL-MIRAJ, M.: "Congenital bladder diverticulum: A rare cause of bladder outlet obstruction in children". J. Urol., 162: 2112, 1999.

5. STEPHENS, D.: "The vesicoureteral hiatus and paraureteral diverticula". J. Urol., 121: 786, 1979.

6. CANNING, D.; KOO, H.; DUCKETT, J.: “Anomalies of the bladder and cloaca". Adult and Pediatric Urology. Vol 3 p. 2445-2488. J. Gillenwater, J. Grayhack, S. Howards, J. Duckett. Mosby, St. Louis, 1996.

7. LINKE, C.; MONGIAT-ARTUS, P.: "Traitement des diverticules vésicaux". Annales d'Urol., 38: 103, 2004.

8. BARRETT, D.; MALEK, R.; KELALIS, P.: "Observation on vesical diverticulum in childhood". J. Urol., 116: 234, 1976.

*9. BOGDANOS, J.; PALEODIMOS, I.; KORAKIANITIS, G. y cols.: "The large bladder diverticulum in children". J. Ped. Urol., 1: 267, 2005.

10. SHUKLA, A.; BELLAH, R.; CANNING, D. y cols.: "Giant bladder diverticula causing bladder outlet obstruction in children". J. Urol., 172: 1977, 2004.

11. VERGHESE, M.; BELMAN, B.: "Urinary retention secondary to congenital bladder diverticula in infants". J. Urol., 132: 1186, 1984.

12. SHELDON, C.; ESSIG, K.: "Congenital bladder diverticulum causing bladder outlet obstruction: Case repport and review of the literature". Pediatr. Surg. Int., 9: 141, 1994.

13. OGE, O.; GEMALMAZ, H.; OZEREN, B.: "Acute urinary retention in a child caused by a congenital bladder diverticulum”. J. Ped. Surg., 37: 926, 2002.
14. MARTÍN-CRESPO, R.; LUQUE, R.; NAVASCUES, J. y cols.: "Alteraciones funcionales de la vejiga en los niños portadores de divertículo vesical primario". Arch. Esp. Urol., 50: 661, 1997.

15. LEVARD, G.; AGRAIN, Y.; FERKADJI, L. y cols.: "Urinary bladder diverticula and the Ehlers-Danlos syndrome in children". J. Pediatr. Surg. 24: 1184, 1989.

16. BADE, J.; YPMA, A.; VANELK, P. y cols.: "A pelvic mass: Bladder diverticulum with haemorrhage in Ehlers-Danlos patient". Scand. J. Urol. Nephrol., 28: 319, 1994.

17. GALIFER, R.; VEYRAC, C.; LÓPEZ, C. y cols.: "Malformations vésicales rares". Encyclopédie médico-chirurgicale 18-208C10. Elsevier, Paris, 2003.

18. JORION, J.; MICHEL, M.: "Spontaneous rupture of bladder diverticula in a girl with Ehlers-Danlos Syndrome". J. Ped. Surg., 34: 483, 1999.

19. MAYNOR, C.; KIEWER, M.; HERTZBERG, B. y cols.: "Urinary bladder diverticula: Sonographic diagnosis and interpretative pitfalls". J. Ultrasound. Med., 16: 189, 1996.

20. PIERETTI, R.; PIERETTI-VAN MARCKE, R.: "Congenital bladder diverticula in children”. J. Pediatr. Surg., 34: 468, 1999.

21. PIRÓ, C.; MARTÍN, J.; CAMPOS, J. y cols.: "Divertículos vesicales primarios. Estudio de 47 casos". Cir. Ped., 2: 191, 1989.

**22. AFSHAR, K.; MALEK, R.; BAKHSHI, M. y cols.: "Should the presence of congenital para-ureteral diverticulum affect the management of vesicouretereral reflux?". J. Urol., 174: 1590, 2005.

23. KOK, K.; SENEVIRATNE, H.; CHUA, H. y cols.: "Laparoscopic excision of congenital bladder diverticulum in a child". Surg. Endosc., 14: 501, 2000.

24. YU, T.: "Extravesical diverticuloplasty for the repair of a paraureteral diverticulum and the associated refluxing ureter". J. Urol., 168: 1135, 2002.

25. SARIHAM, H.; ABER, M.: "Congenital bladder diverticula in infants". Eur. Urol., 33: 101, 1998.

26. GONZÁLEZ, J.; RODÓ, J.; ARZA, H. y cols.: "Divertículos vesicales en el niño”. Rev. Esp. Pediatr., 45: 54, 1989. 